\title{
Ethnographies du design
}

Vers une diagrammatisation de la conception

\section{Vincent Beaubois}

\section{(2) OpenEdition \\ Journals}

\section{Édition électronique}

URL : https://journals.openedition.org/tc/7558

DOI : $10.4000 /$ tc. 7558

ISBN : 0248-6016

ISSN : 1952-420X

Éditeur

Éditions de l'EHESS

\section{Édition imprimée}

Date de publication : 24 décembre 2015

Pagination : 48-63

ISBN : 978-2-7132-2505-5

ISSN : 0248-6016

Référence électronique

Vincent Beaubois, «Ethnographies du design », Techniques \& Culture [En ligne], 64 | 2015, mis en ligne le 24 décembre 2018, consulté le 29 septembre 2022. URL : http://journals.openedition.org/tc/7558 ; DOI : https://doi.org/10.4000/tc.7558 


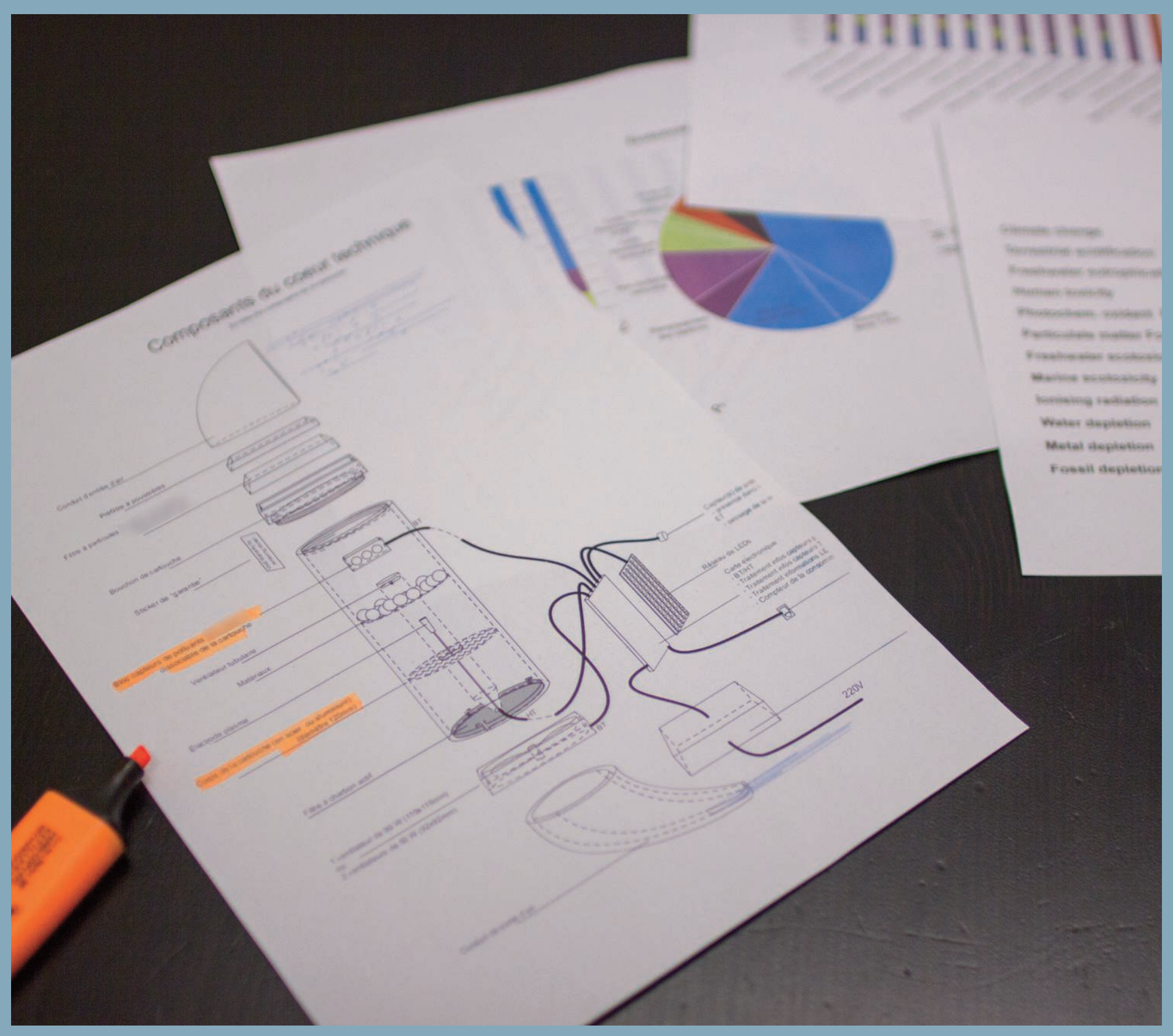




\section{Ethnographies du design Vers une diagrammatisation de la conception}

Si l'ethnologie et la sociologie des techniques et de l'architecture s'intéressent de façon marquée aux pratiques de conception des artefacts depuis la fin des années 1990 (Callon 1996; Houdart 2006; Yaneva 2009), il faut prendre la mesure de cet apport pragmatiste sur la compréhension du design comme activité de création ${ }^{1}$. Il s'agit de réinvestir la question d'un pragmatisme du design, entendue comme processus de prise de forme des artefacts (objets, images, dispositifs, etc.), à la lumière des philosophies contemporaines s'intéressant à la production matérielle comme genèse (Gilbert Simondon, Félix Guattari et Gilles Châtelet), afin d'éclairer de façon nouvelle la pensée propre à cette pratique, une pensée investissant la matière comme matière à inventer.

Prenons un exemple tiré de la conception architecturale: le projet signé par l'architectedesigner Andrea Branzi concernant le Tokyo International Forum, lieu d'expositions, de conférences et de spectacles, défini en collaboration avec Franco Lani et Tullio Zini en 1989. Comment rendre compte de cette production, d'autant que le projet n'a jamais été «réalisé», n'ayant pas remporté l'appel d'offres de la préfecture de Tokyo? Ce qu'il reste de cette forme en suspens, ce sont les différentes images participant de ce processus de création. L'ensemble du projet, tel qu'on peut l'observer dans les archives du Centre Pompidou (Guiheux 1998), se présente sous la forme d'une multiplicité d'éléments hétérogènes: esquisses, plans, perspectives in situ, maquettes volumiques, etc. Un texte présente l'intention architecturale. Cette «intention » prend acte d'un paysage métropolitain tokyoïte fragmenté, aux perspectives sans cesse interrompues et sans aucune hiérarchie sémantique - «a never ending hybridation ». Branzi insiste sur l'importance de dessiner une figure reconnaissable exprimant la sensibilité nouvelle imposée par ce cadre de vie artificiel. C'est en cela qu'une forme d'oreille colossale domine l'édifice, abritant un réseau d'antennes et de réception satellite, comme le centre d'une mégapole devenue un gigantesque système d'informations. 
Or, quel sens donner à ce travail, dans les deux acceptions du terme? Quelle direction la prise de forme suit-elle? Doit-on partir de l'intention formulée pour comprendre la mise en forme du bâtiment? Peut-on dire que c'est l'intention première de faire de ce bâtiment le nœud d'un réseau d'informations qui a conduit à la prégnance de cette forme d'oreille? Et peut-on affirmer que c'est l'intention de formaliser ce signe-oreille qui a engagé le bâtiment à prendre sa forme de socle monolithique surmonté d'une antenne géante?

La question simple que nous souhaiterions poser concerne donc la place de cette «intention» dans le processus de conception, ou plus précisément : comment définir la «finalité » qui travaille la chose en conception? Il existe en fait deux manières classiques et antagonistes de penser le processus de conception. John Christopher Jones résume cette dissension par l'image de la boîte noire (black box) et de la boîte transparente (glass box):

«Du point de vue créatif, le designer est une boîte noire d'où découle le mystérieux élan créatif; du point de vue rationnel, le designer est une boîte transparente à l'intérieur de laquelle peut être discerné un processus rationnel entièrement explicable².» (Jones 1970: 46)

D'un côté, la forme la plus aboutie du bâtiment de Branzi serait une excrétion opaque issue des méandres de son espace mental: l'artefact vient matérialiser et représenter le concept architectural établi au préalable par son géniteur. De l'autre, le Tokyo International Forum serait le produit d'étapes logiques et progressives que l'on pourrait reconstruire a posteriori: en analysant les différentes esquisses produites durant le projet, on peut saisir les différents choix formels et techniques successifs aboutissant à l'œuvre que nous connaissons. Ces deux options, en apparence opposées, postulent la maîtrise intentionnelle des « créateurs » guidant le projet vers sa réalisation. Elles partagent également un même préjugé. Ce préjugé commun tient à une double analyse se constituant depuis l'objet déjà là, déjà achevé, repensant la création a posteriori, à la manière de la génétique des œuvres littéraires, cherchant à éclairer la constitution d'un écrit consacré à l'aide de ses brouillons et manuscrits. Or, le problème ne tient pas à la couleur de la boîte mais à l'image même de la boîte statique pour penser le devenir d'un artefact en train de se faire.

\section{Un pragmatisme du design}

C'est ce préjugé que se sont attachés à détruire les travaux ethnographiques infiltrant le processus même de genèse des artefacts: il ne s'agit plus d'interpréter des formes existantes, mais de suivre l'activité de conception se faisant. Cette approche ethnographique du design s'est développée à la fin du xxe siècle dans le sillage des Science and Technology Studies (STS) qui se sont amorcées avec l'observation des pratiques scientifiques en laboratoire ${ }^{3}$. Suivant une démarche parallèle, l'ethnologie pragmatiste appliquée au design s'intéresse à la pratique de conception des artefacts, installant ses terrains d'étude au sein même des agences de design et d'architecture. On peut, à ce titre, noter l'importance des travaux singuliers d'Albena Yaneva (2009) au 
sein de l'agence OMA de Rem Koolhaas, ceux de Sophie Houdart au cœur de l'agence de Kengo Kuma (Houdart \& Minato 2009), ou encore l'analyse proposée par Michel Callon (1996) des travaux de Francis Rathier et Guy Tapie sur les processus de conception en architecture. Le mot d'ordre à l'initiative de telles études s'exprime simplement: «rien ne peut être dit du fait (d'un bâtiment ou d'une exposition) sans avoir le faire (la préparation, les outils du travail du bâtiment, de l'exposition)» (Houdart \& Minato 2009: 28). Contre une perspective idéaliste, il faut d'abord filer le projet dans la matérialité des images qu'il produit.

Car, que nous révèlent ces terrains d'études? Ils manifestent, en premier lieu, ce qui peut apparaître comme une belle évidence quant à la nature du travail de conception : le design ne produit pas d'objets. Que produit une agence de design ou d'architecture, littéralement? Certainement pas des objets, au sens d'objets tangibles, définitifs, prêts à l'emploi, artefacts ou bâtiments, mais plutôt ce que nous pourrions désigner comme des «images», pour qualifier tous ces intermédiaires que sont les esquisses, les infographies et autres maquettes. Une agence de design ne fait que produire des images prototypant l'objet à venir par des opérations de mise à l'échelle, de test fonctionnel, de simulation, de choix de textures, etc. «Image» ne doit pas s'entendre au

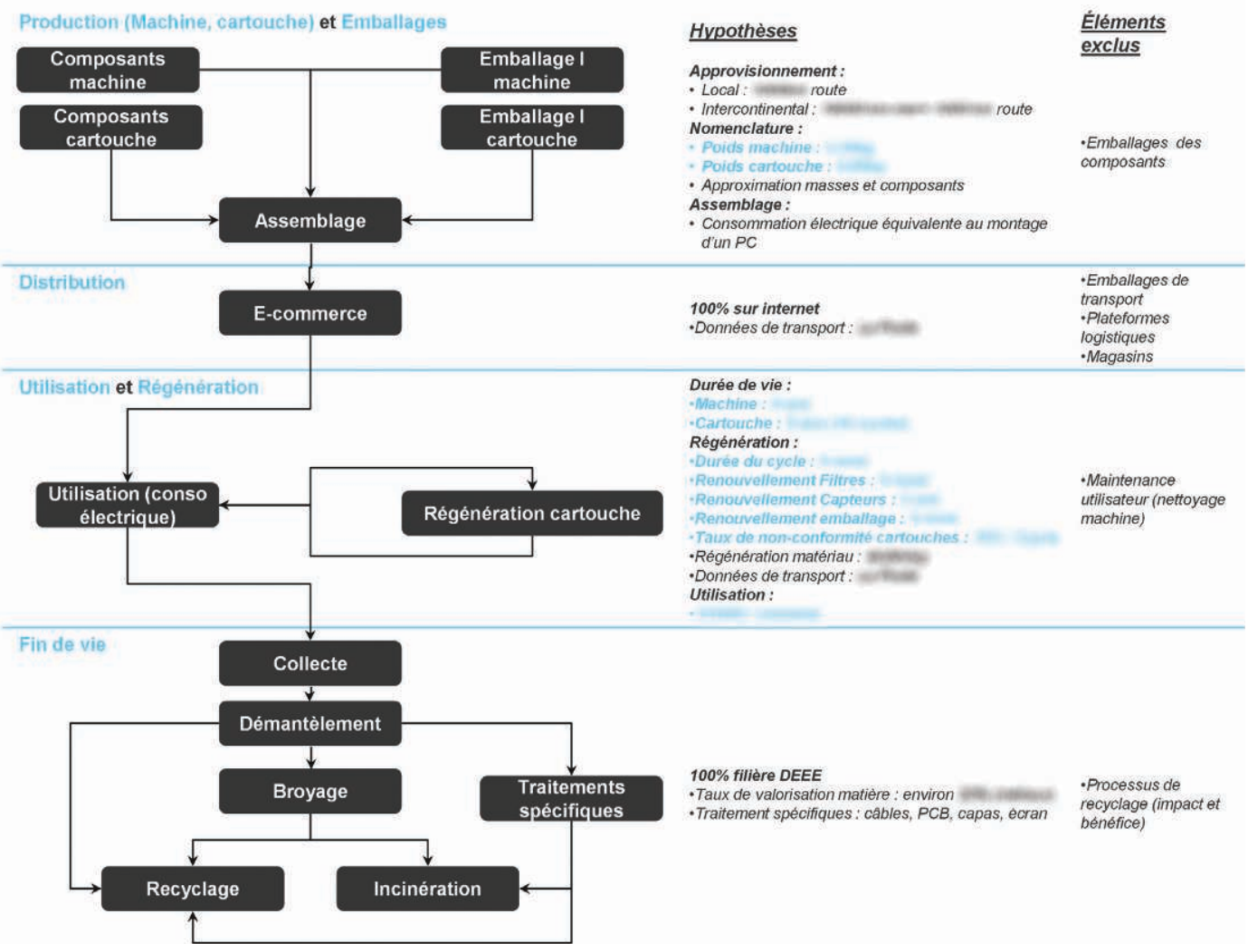

Exemple d'image de conception: diagramme de cycle de vie d'un produit électroménager lié à la santé. 
sens de « représentation » graphique d'un original qui serait d'abord mental mais au sens que lui confère Gilbert Simondon dans son cours de 1965, Imagination et invention. Simondon développe, dans ce cours, une théorie originale de l'image: celle-ci ne doit pas d'abord être considérée comme un pur produit de la conscience, insistant au contraire sur sa «relative indépendance [...] par rapport au sujet» (Simondon 2008: 9). Les images «apparaissent presque comme des organismes secondaires au sein de l'être pensant: parasites ou adjuvantes, elles sont comme des monades secondaires habitant à certains moments le sujet et le quittant à certains autres » (ibid.). Les artefacts eux-mêmes jouent le rôle de réservoir d'images, apparaissant ainsi comme de véritables «objets-images». Par leur forme, leur couleur, leur fonctionnement, leur texture, leur matériau, ils instancient un réseau de signes polarisant potentiellement les attentes et les désirs de celui qui les pratique:

«Presque tous les objets produits par l'homme sont en quelque mesure des objets-images; ils sont porteurs de significations latentes, non pas seulement cognitives, mais aussi conatives et affectivoémotives; les objets-images sont presque des organismes» (ibid. : 235).

L'objet-image caractérise non un simple objet statique, mais un objet présentant une charge potentielle pouvant mettre en mouvement l'action ou la pensée de celui qui l'éprouve. Prendre la

Objet-image numérique: modélisation sous SimaPro ${ }^{\circledR}$ des impacts environnementaux de cet objet.

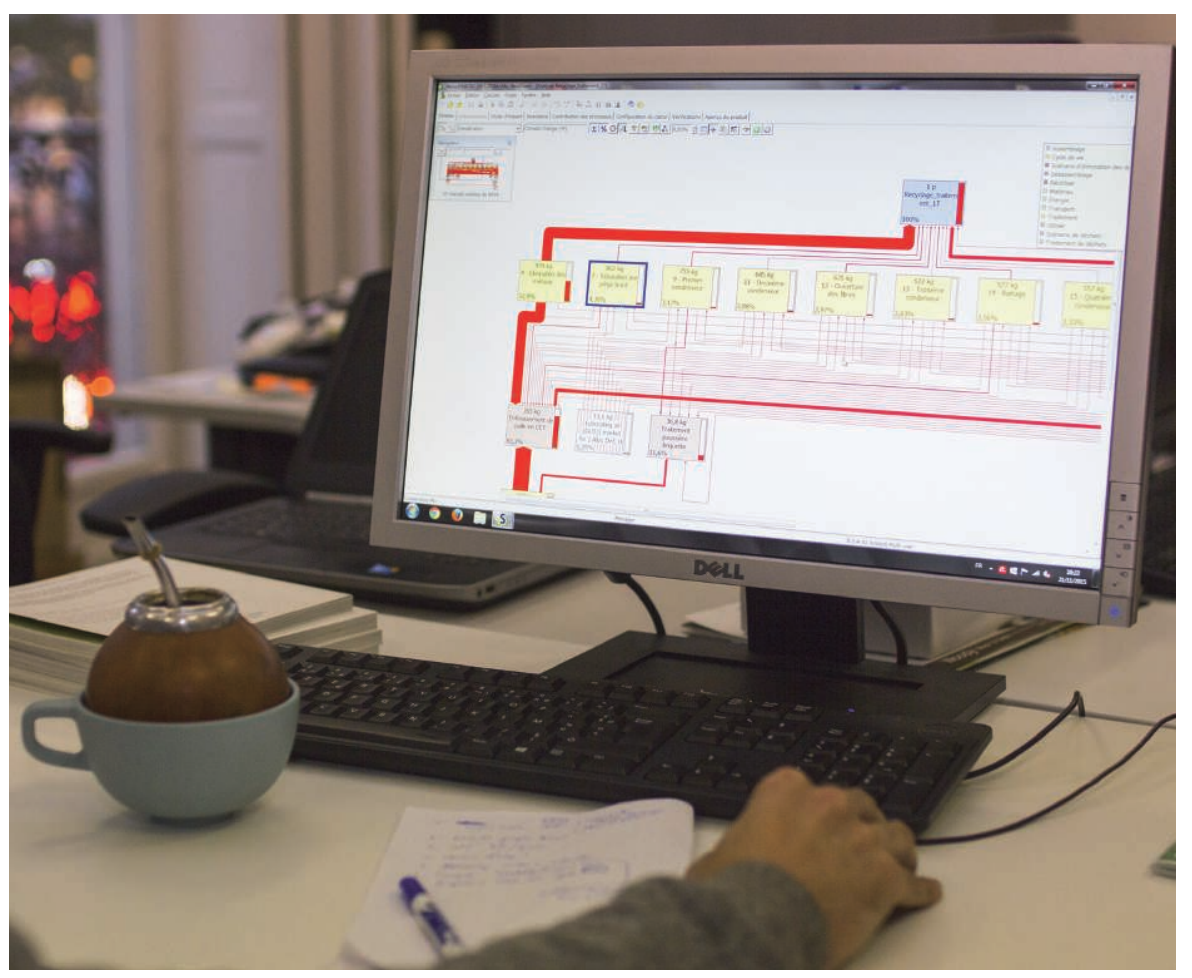


mesure de ces objets-images que sont les maquettes et les prototypes, c'est rendre compte du potentiel qu'ils expriment sur la pensée conceptrice. Une maquette volumique ou un prototype fonctionnel ne sont pas de simples « représentations» tridimensionnelles mais plutôt des agents d'individuation d'un projet, de même qu'un plan technique ou une infographie. Ces images et objets-images, définissant l'espace du projet design, s'entr'expriment et se transforment les uns dans les autres, étant sans cesse repris, retravaillés et mis en série. Ce réseau d'images développe ainsi une dynamique pouvant faire émerger des problèmes de conception, qualifier des champs de contraintes ou valider des hypothèses. Il rend possible une pensée conceptrice et inventive, ces images apportant « la réserve de leur pouvoir et de leur savoir implicite au moment où des problèmes doivent être résolus» (ibid. : 27).

Albena Yaneva affirme ainsi «suivre les infimes opérations de modélisation» de la conception afin d'éclairer la manière qu'ont les architectes, les designers et les techniciens d'acquérir une connaissance sur le projet dans lequel ils sont pris, notamment lors de son étude du projet d'extension du Whitney Museum à New York par l'agence OMA en 2001 :

«Non seulement les maquettes au sein d'OMA "font des déclarations", mais elles appellent les questions ou y répondent, donnent des informations, formulent des hypothèses, expriment des vœux, rassurent ou alertent, créent des anticipations, annoncent des intentions. [...] Elles agissent comme
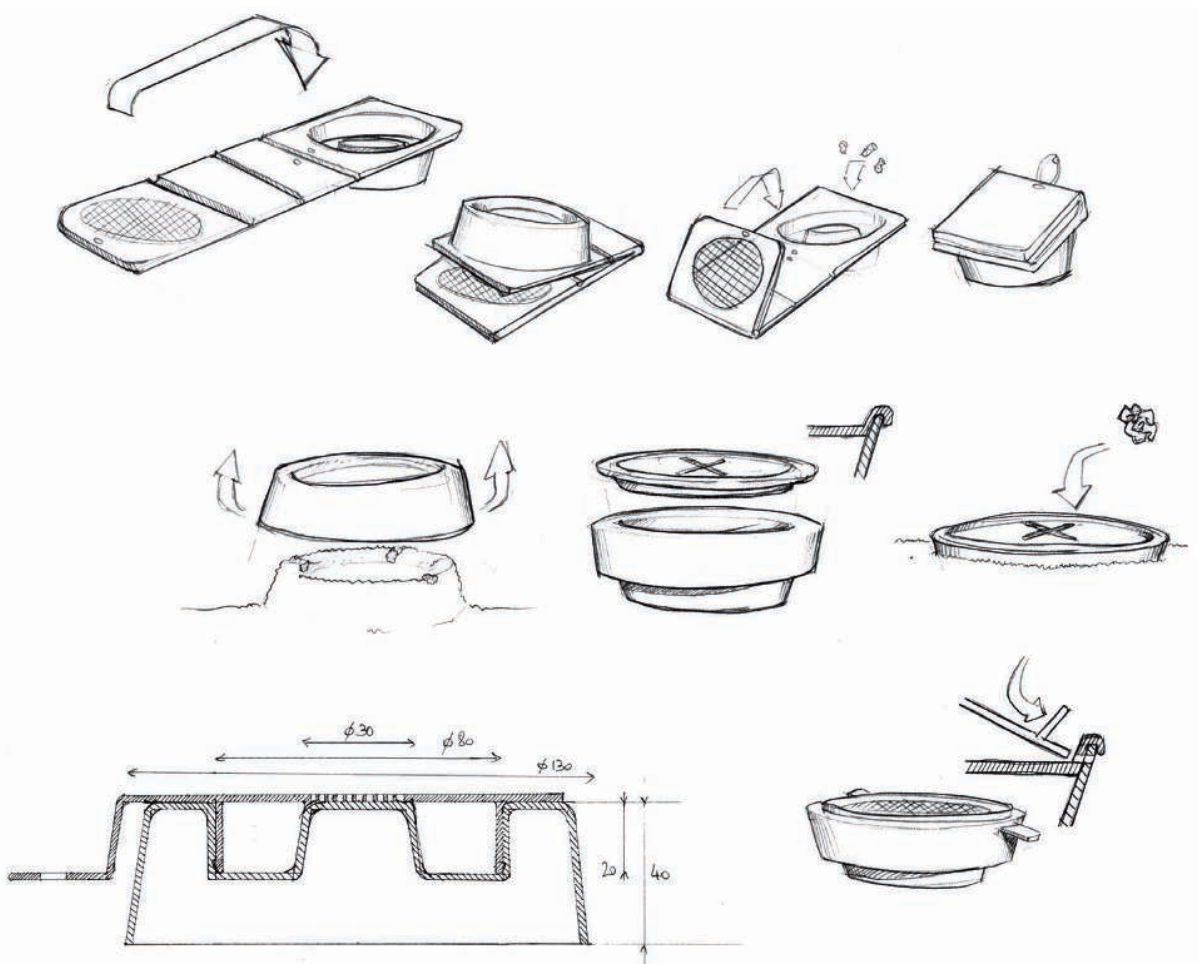

Croquis de conception cherchant à synthétiser des contraintes de montage, d'assemblage et d'usage d'un produit. 
autant d'outils d'investigation gestuels, spatiaux et opérationnels, aidant les architectes à en apprendre plus du Whitney et à interpréter ses fonctions. C'est en engageant un dialogue avec des matériaux concrets, des figures spatiales, des proportions, des dispositions et des formes, que les designers de l'agence OMA acquièrent toujours plus d'informations sur le NEWhitney. » (Yaneva 2009a: 120, notre traduction)

Faisant éclater l'opposition entre une matérialité brute d'un côté, et un supplément idéel ou symbolique de l'autre, le terrain ethnographique entend rendre compte des formes artefactuelles sans recours à de grands signifiants comme «société», «culture» ou «imagination », qui n'expliquent rien mais appellent plutôt une explication (ibid. : 28). Suivre le processus de conception dans ses constructions matérielles et la pensée qui en découle nous évitent ainsi d'ajouter cette dimension symbolique ou intentionnelle pour expliquer la construction matérielle. L'approche ethnographique prend le pli de cette production d'objets-images, privilégiant une approche myope, scrutant les détails de la pratique conceptrice. Il s'agit de rendre compte de la création par ces images et à partir de ces images plutôt qu'à partir des intentions des architectes et designers. La conception apparaît alors, non pas comme ce qui s'oppose à la production (comme l'idée pure s'opposerait à la matière pure), mais comme une intense phase de production.

Designer ne consiste plus alors à ajouter du sens à une matière technique brute mais à s'installer dans la matérialité de chaque projet, de chaque esquisse ou maquette dans la mesure où ces objets-images génèrent eux-mêmes du sens. L'invention ne s'explique plus par le génie créateur: "L'invention se produit à partir d'un processus "prenant les maquettes au sérieux", expérimentant et exprimant au moyen de celles-ci, et utilisant divers autres outils de manière originale» (ibid. : 44).

Ces ethnographies du design nous éloignent ainsi de trois préjugés classiques concernant la conception des artefacts: le préjugé de la représentation, faisant des esquisses et autres maquettes de simples illustrations imparfaites de l'objet projeté; le préjugé de l'inertie intrinsèque de l'œuvre spatiale, jugée comme statique par opposition au dynamisme de l'œuvre temporelle; le préjugé du symbolique comme explication juxtaposée à la matière pour rendre compte de son sens.

Par ailleurs, cette intensification de la production des objets-images ne peut se comprendre sans évoquer l'organisation capitaliste qui la sous-tend. Cette prolifération d'images se constitue au sein d'une organisation du travail de conception favorisant la pratique des concours pour exalter la compétition entre équipes de projet. Le concours de design ou d'architecture impose alors des formes de présentation toujours renouvelées pour donner à comprendre le bâtiment ou l'objet conçu en un temps court. Cette multiplication des objets-images crée une mise en spectacle de la création et une esthétisation du faire, vendre un projet consistant alors à le présenter. 


\section{Diagrammatisation de la conception}

La conception prend consistance à partir de la simultanéité de ces différents objets-images, qui partagent, à travers ce réseau, l'idée de l'artefact en train de se faire. Qu'entendons-nous en affirmant que ces objets-images que sont les prototypes, les dessins, les scénarios d'usage partagent l'idée de la chose en conception? Quelle théorie de l'«idée» une telle pratique mobilise-t-elle, dès lors qu'on écarte tout recours à une intention humaine préalable à la conception de l'objet? Une idée ne doit pas se comprendre comme le corrélat spontané d'une conscience clairvoyante, mais comme se pensant de proche en proche, au fur et à mesure des versions matérielles testées et approfondies. Comment alors rendre compte de cette idée se faisant sans en faire ni un contenu mental ni un simple résidu matériel?

L'idée d'une chose en conception est ce qui conduit sa construction, tout en s'effectuant dans sa prise de forme. Qu'il y ait de l'idéel dans le design, on ne peut en douter. Une maquette présentée lors d'un rendu final d'architecture est un être double dès l'instant que le spectateur

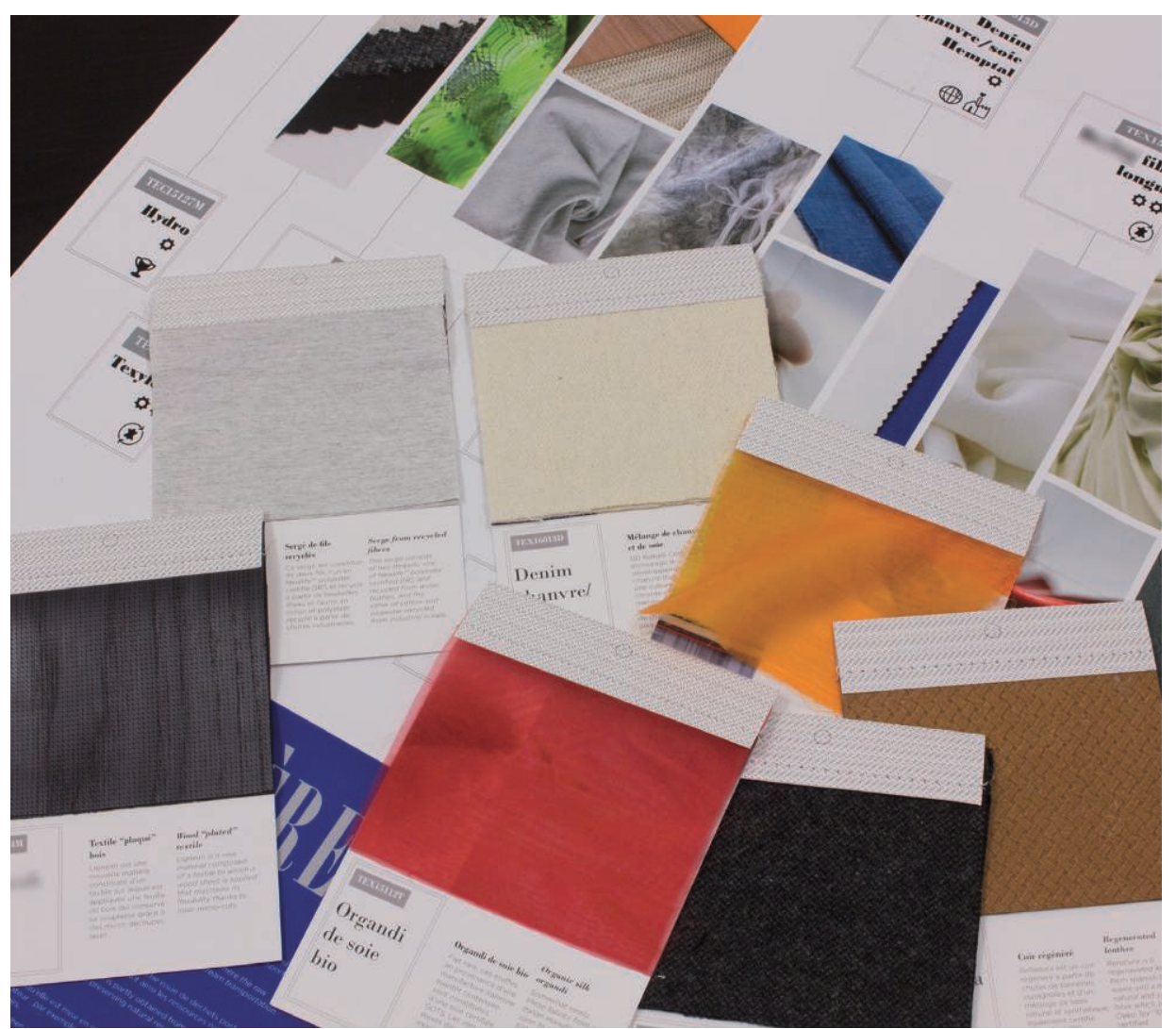

4. Matériauthèque composée d'un ensemble d'échantillons fonctionnant comme autant d'objets-images dans le choix d'un matériau. 
qui la reçoit ne peut distinguer «ce qui, dans l'image, tient du réel, du déjà construit, du déjà actualisé, et ce qui est du registre de la projection, du non encore advenu » (Houdart 2006: 121122). La maquette et le bâtiment final se superposent dans l'œil du spectateur: ce dernier voit, littéralement, le bâtiment construit. Ainsi, Sophie Houdart précise que l'effet rate si le dessin ou la maquette ne peuvent se passer du discours de son producteur pour être convaincants. Une infographie qui ne parvient pas, par elle-même, à exprimer le rendu d'un bâtiment échoue:

«L'image de Sakakura est une image ratée parce que c'est une image qu'on voit - plus précisément, c'est l'image elle-même qu'on voit et non ce à quoi elle réfère. [...] Le dessin, autrement dit, doit pouvoir parler de lui-même et se passer d'interprète. Il ne doit pas avoir besoin de quelqu'un pour expliciter la fameuse "intention architecturale" (ibid. : 122).

L'idée du bâtiment ne recouvre pas la notion d'«intention». Au contraire, le recours à l'intention dénote l'échec de l'idée, échec de l'objet-image à instancier l'idée. C'est en cela qu'un objet-image peut échouer, pouvant être à la fois trop matériel (on ne voit que le support) et trop idéel (nécessité de faire intervenir une intention). Une bonne maquette n'est pas tant la plus réaliste que celle qui donne à penser l'idée de l'artefact en conception, celle qui met la pensée en mouvement : elle montre ce qui reste à faire, à développer, à transformer.

Le design travaille dans et par ces images mêmes. Si bien, qu'en suivant un terme de Félix Guattari, on peut parler du design comme d'une activité diagrammatique. Pour Guattari, le régime du « diagrammatique » sert à caractériser l'état d'une chose qui est en transformation ou en genèse. Un exemple récurrent, qu'il emploie pour faire comprendre ce régime d'expérience, concerne le mode d'existence d'une particule au vu de la microphysique contemporaine: la particule n'existe jamais à partir d'une expérience directe, mais nécessite toujours un complexe «théorico-expérimental» fait d'équations mathématiques, d'accélérateur de particules, de personnel scientifique, de calculateurs, etc., pour trouver une consistance. La particule n'existe donc qu'à travers ce « diagramme»; elle est « virtualisée» par ce diagramme (Guattari 2011 : 168). De la même façon, une chose en conception - c'est-à-dire ce sur quoi travaillent les designers dans le temps de leur pratique et qui n'est pas encore défini puisque tout leur travail consiste à définir cette chose: que ce soit un objet, une image, un dispositif, une interface, etc. n'existe d'abord que de manière virtualisée par un dispositif diagrammatique constitué de ces images que sont les prototypes, les plans, les croquis, les procédures, etc.

Cette genèse de l'objet se consolidant à partir de différents matériaux hétérogènes est également qualifiée par le designer Ettore Sottsass de «diagramme» (Bischofberger 1995: 10). Le diagramme s'oppose à l'objet statique. Relatant son expérience en Inde dans les années 1960 et l'influence de la Beat Generation sur son travail, Sottsass met en avant l'importance de ce «système ouvert » en devenir comme pratique du design:

«Je nourrissais déjà un certain intérêt pour les cultures orientales, en particulier pour celle de l'Inde, et avec Ginsberg je trouvais des confirmations: la possibilité d'une translation, la possibilité d'imaginer l'existence d'une manière différente que ne le faisait l'Occident. Non pas comme un calendrier 
rigide avec des objectifs à atteindre, mais comme un système ouvert qui se renouvelle chaque jour. Cette façon de penser est issue d'une attitude détachée et simple, mais aussi très intense. Il se peut que certaines choses que nous avons faites soient presque des diagrammes, des représentations de ces idées.» (Grandi 2011, notre traduction)

Sottsass insiste ainsi sur le fait que le design se constitue par diagrammes, certaines de ces productions ayant été travaillées par des images littéraires issues de la Beat Generation ou de ses voyages en Inde dans les années 1960.

Le design consiste alors en une configuration diagrammatique d'images qui mettent au travail les différents métiers qui lui sont liés (styliste, modeleur, infographiste, etc.). À la croisée des pensées de Gilbert Simondon et de Félix Guattari, le travail de Gilles Châtelet s'avère particulièrement intéressant pour saisir l'importance de cette pensée diagrammatique de la conception, ayant lui même fait fonctionner cette notion dans sa compréhension d'une pratique

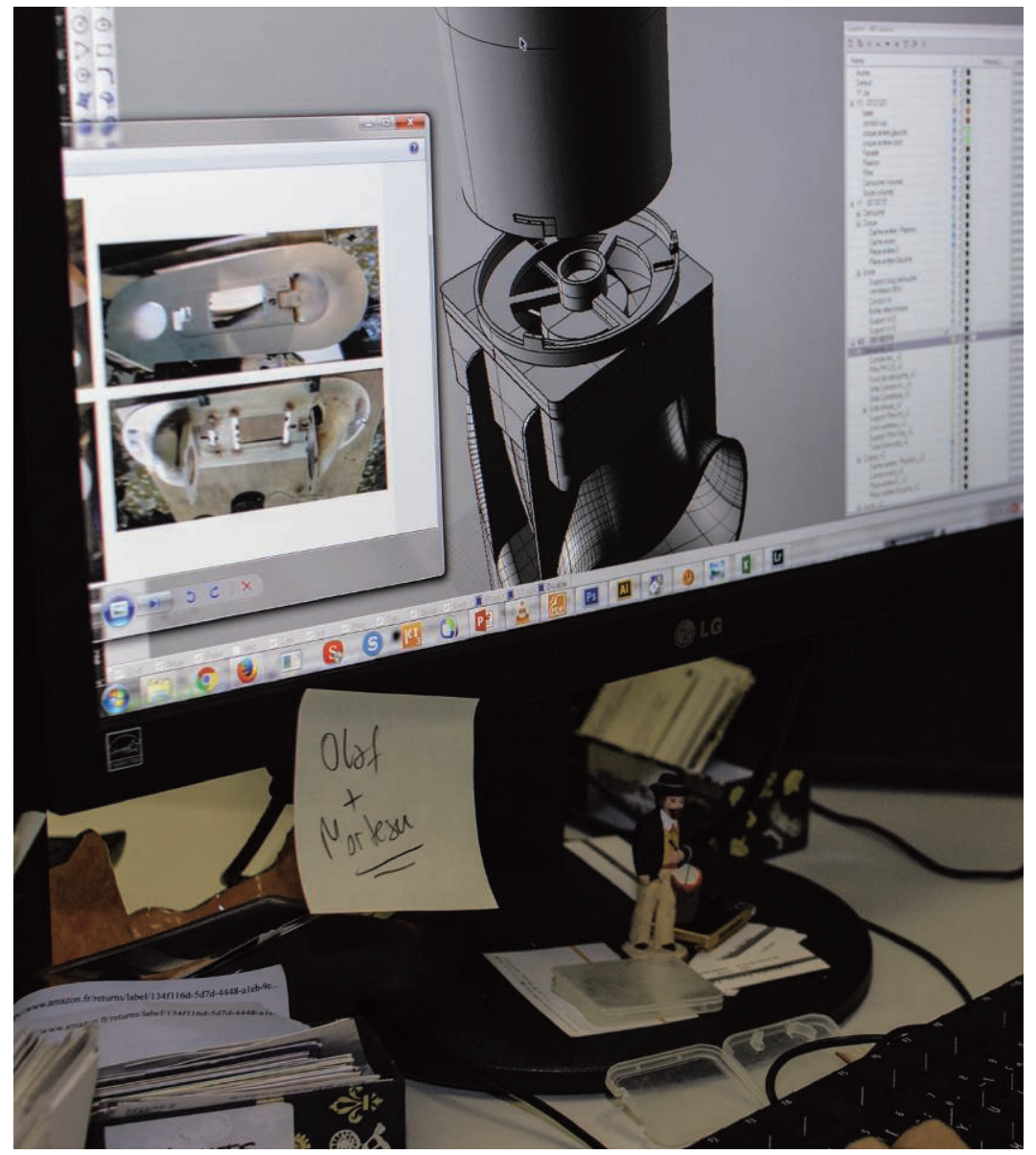

Reprise d'une modélisation numérique suite à la production d'un prototype fonctionnel. 
humaine appelant créativité et invention: les mathématiques ${ }^{4}$. Penser par diagramme, c'est se défaire du simple rapport de symbolisation de l'idée au matériau pour penser une imbrication, une articulation de l'idéel et du matériel. Le terme même de «diagramme» inscrit dans sa lettre cette dualité, associant le préfixe «dia» marquant un processus, une traversée, et le "gramme» indiquant une inscription pouvant se faire lettre ou image ${ }^{5}$. Dans cette perspective, le diagramme apparaît à la fois comme manifestation du «gramme» et de ce qui le traverse, l'emportant ailleurs, par-delà sa simple matérialité.

Selon la typologie sémiotique de Charles Sanders Peirce, le diagramme désigne une forme d'«icône de relation », c'est-à-dire d'image ayant pour but de représenter les relations entre des parties formant un tout. Le diagramme n'est pas simplement représentatif puisqu'il permet au contraire de produire des informations à partir de ces mises en relations (Peirce 1978: 150). Une carte, par exemple, est un diagramme en tant qu'elle renvoie à une multiplicité de relations entre une topologie géographique, une distribution des espaces urbains, des réseaux de routes, etc. Faisant écho au terme de Peirce, Châtelet insiste sur sa dimension productive: le diagramme n'est pas simplement représentation de relations mais producteur de relations.

Châtelet interroge le rôle cognitif des configurations spatiales, soutenant ainsi que la réflexion sur les mathématiques doit inclure une compréhension « des technologies manuelles dont nous nous servons pour représenter l'espace, au moyen de marques, de dessins, de croquis, de gribouillis, etc.» (Knoespel 2004: 144). Comme le précise Kenneth Knoespel, ces images sont d'abord «le site d'un travail d'invention et de découverte dont les théorèmes sont impuissants à rendre compte, tant ils verrouillent notre compréhension des procédures mathématiques» (ibid.). Prenant appui sur l'usage du diagramme en mathématiques (diagrammes de Feynman, de Penroe, d'Argand ou diagrammes d'espace-temps de Minkowski), Châtelet cherche à montrer que le diagramme s'efforce d'articuler une compénétration de l'image et du calcul. Le diagramme désigne ainsi le schéma que le mathématicien trace pour «travailler » une idée mathématique. Il ne s'agit pas d'une simple illustration de l'idée mathématique, mais bien de cette idée même en acte. L'opération inscrite dans le tracé permet d'instituer des expériences de pensée qui la raccordent à l'être même de l'idée mathématique. Comme l'indique Charles Alunni dans son introduction à l'Enchantement du Virtuel, recueil de textes de Châtelet: "le diagramme mathématique rend observables des relations autrement invisibles. [...] [il] rend ainsi familier, concret et sensible à l'œil de l'esprit ce qui pourtant relève purement de l'ordre du contre-intuitif» (Alunni 2010: 44-52).

Le diagramme d'Argand, par exemple, en montrant simplement que l'unité «imaginaire » des nombres complexes $i$ n'est rien d'autre qu'une rotation de $\varpi / 2$, opère pour la pensée une relation riche de potentiel entre la théorie algébrique et la théorie géométrique. Le diagramme instaure un espace au service de l'intuition mathématique: « une philosophie du physico-mathématique ne saurait ignorer cette pratique symbolique en amont du formalisme, pratique de condensation et d'amplification d'intuition » (Châtelet 1993: 34). Le diagramme opère par-delà a priori et a posteriori: 
"Cette "vue d'ensemble" n'est pas une contemplation distante de dilettante; elle participe à l'action: c'est une intuition intellectuelle, au sens où l'entend la philosophie de la nature. Elle nous transporte dans cette zone privilégiée où intuition et discursivité nouent une vivante unité. Elle n'est ni a priori ni a posteriori; elle est contemporaine de ce qu'elle saisit » (ibid. : 159).

Le diagramme rend possible l'anticipation de processus potentiels du fait de son caractère opératoire: « lire un diagramme correctement, c'est le faire fonctionner. Ce fonctionnement, qui l'oppose à une représentation statique et inerte, n'est possible que parce qu'il est investi par des virtualités internes ${ }^{6} »$ (Bontems 2011: 103).

Le diagramme est ainsi un outil d'anticipation pour la pensée conceptrice. Le design ne s'instancie pas dans une maquette ou dans un prototype, mais dans des séries, dans un réseau d'objets-images composant le projet. C'est ce réseau qui fonctionne comme diagramme. Le diagrammatique dans la conception désigne ainsi l'ensemble des traits différentiels entre objetsimages venant exprimer ce qu'est la chose à concevoir selon ses différentes dimensions: techniques, pratiques, esthétiques, etc. C'est en tant qu'il instancie l'idée virtuelle de la chose en train de se faire que l'objet-image possède un potentiel de reprise. La maquette dit toujours:

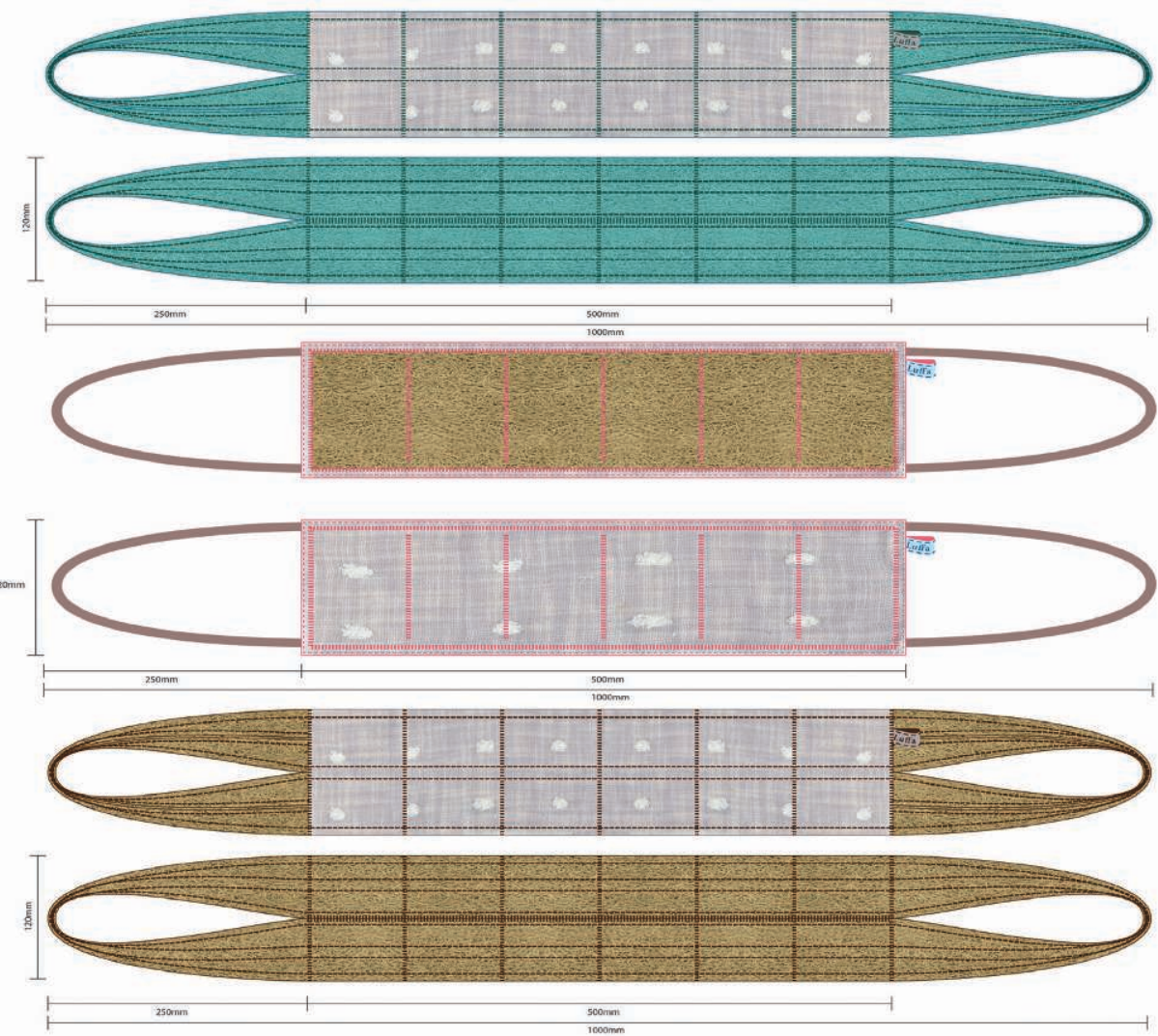

6. Comparaison, dans un même plan de définition, de différents produits en luffa (éponge végétale). 
«je suis là mais il y a encore plus à faire» (Yaneva 2009b: 61), appelant, de manière continue, la reprise et la transformation de son idée. Le lien causal génétique entre deux objets-images, entre une maquette et une infographie par exemple, est une cause qui ne s'actualise que dans son effet, elle s'intègre dans son effet génétique, elle se différencie comme cause dans cet effet. Si bien qu'on ne sait ce qui était virtuellement présent dans la maquette qu'une fois l'infographie réalisée. Cette actualisation donne ainsi cours à une forme matérielle et à une nouvelle caractérisation signifiante de cette forme. Le diagramme, c'est l'idée même de la chose se faisant dans un réseau d'objets-images matériels. La conception se fait en diagrammatisant, tel le chemin d'Antonio Machado: «Caminante no hay camino, se hace camino al andar ${ }^{7}$ » (Machado 2012: 130). Le diagramme n'est pas inscription passée, mais orientation vers l'avenir, anticipant et faisant advenir. La conception comme diagrammatisation de la forme est moins une démarche de résolution de problème qu'une exploration et une configuration de multiplicités problématiques.

Si des finalités humaines participent au projet, elles n'en ont pas l'initiative, mais elles se raccordent à lui : la finalité à l'œuvre dans la conception se redéploie en permanence dans la fréquentation de ce milieu d'objets-images matériels en constitution. Ainsi, le diagramme n'est pas une simple icône illustrant une intentionnalité, mais, au contraire, ce qui permet de s'éloigner des intentions subjectives premières pour rendre possible l'invention. La conception est diagrammatique en tant qu'elle est une coopération entre des images qui font inventer et des humains inventant. Comme le dit Yaneva elle-même, les maquettes sont des objets de «provocation de la pensée» (thought provocation) (ibid. : 56). En se faisant, le diagramme existe toujours selon deux phases, graphique et actuelle d'un côté, idéelle et virtuelle de l'autre. Cette différenciation, produite par l'acte même de conception, explique alors l'illusion du dualisme matière/ idée qui vient hanter les définitions conventionnelles de la création dès lors qu'on s'éloigne d'une approche pragmatiste de la conception pour considérer uniquement ses produits achevés.

En privilégiant cette approche myope que nous offre l'analyse ethnographique, scrutant les détails de la conception dans ses moindres images en train de se faire, c'est la compréhension traditionnelle du design, comme processus linéaire partant de l'esprit du designer jusqu'à la prise de forme dans la matière, qui se trouve contredite. Lactivité de projet agit au contraire toujours comme une coopération entre des humains pensant et des objets-images qui font penser, dans l'individuation d'une idée se concrétisant par différents matériaux. La relation entre conception et production se trouve inversée: c'est la production (des objets-images) qui sert la conception, et non l'inverse. Production et conception entrent en relation réciproque dans une diagrammatisation de la forme. 


\section{Notes}

1. Je souhaite vivement remercier la Coopérative $\mathrm{Mu}$ (François-Xavier Ferrari, Anthony Boule, Florent Chalot), agence d'éco-conception à Paris, pour leur accueil et pour m'avoir autorisé à photographier et collecter différents diagrammes liés à leurs projets de conception. Ces images ne sont pas une simple illustration du texte. Celui-ci, commentant des exemples liés aux auteures discutées (Sophie Houdart et Albena Yaneva), ne reprend pas directement les projets photographiés. Ainsi, le texte et les images forment deux séries autonomes développant ce qui se joue dans un processus de conception. Ces séries entrent alors en résonance autour de deux concepts clés articulant texte et image: "diagramme» et «objet-image».

2. Notre traduction, nous soulignons. Jones défend l'approche «glass box»; Albena Yaneva reprend cette typologie en identifiant d'autres théoriciens de la boîte transparente (Yaneva 2009: 119)

3. Il s'agit alors d'informer la constitution des faits scientifiques à travers l'ensemble des opérations d'expérimentation et de visualisation du travail des chercheurs. Héritiers du pragmatisme de William James, les ethnologues et sociologues s'intéressent alors non pas aux résultats de la science mais aux processus de genèse de ces résultats (Latour \& Woolgar 1979; Lynch 1993).

4. L'intérêt de Châtelet pour le concept de «diagramme» tient à l'importance de cette notion dans les travaux de Guattari seul (Guattari 1979) et avec Deleuze (Deleuze \& Guattari 1980), ainsi que de la lecture que fait Gilles Deleuze de la peinture de Francis Bacon (Deleuze 1981) et de la pensée de Michel Foucault (Deleuze 1986). Gilles Châtelet hérite à la fois de cette pensée du diagramme et de la pensée génétique de Simondon.

5. Noëlle Batt précise ainsi qu'à l'origine de « gramme», on trouve l'association de deux racines indo-européennes, "grbh» et «mn», où la première signale l'action de gratter, qui engendrera ensuite le fait de tracer, dessiner, écrire mais aussi le crabe, qui inscrit ses déplacements dans le sable, ainsi que la gravure incisant le bois, la pierre ou le cuivre, et la seconde, «mn», donnera naissance à image, lettre, texte (Batt 2004: 6).

6. Dans cet article, Vincent Bontems souligne très justement la nature diagrammatique de la pensée simondonienne.

7. « Toi qui marches, il n'existe pas de chemin, le chemin se fait en marchant».

\section{L'auteur}

Vincent Beaubois est agrégé de philosophie et doctorant à l'Université Paris X (Nanterre). Il travaille sur des questions de philosophie de la technique, de design et d'esthétique. Sa thèse porte sur la question de la conception des objets au prisme de la pensée de Gilbert Simondon.

\section{Iconographie}

Image d'ouverture. Série de diagrammes de conception d’un produit électroménager lié à la santé.

\section{Références}

Alunni, C. 2010 «Des Enjeux du mobile à L'Enchantement du virtuel - et retour » in G. Châtelet L'Enchantement du virtuel: mathématique, physique, philosophie, Paris: Éditions Rue d'Ulm: 7-60.

Batt, N. (dir.) 2004 Penser par le diagramme de Gilles Deleuze à Gilles Châtelet, Paris: Presses universitaires de Vincennes ( Théorie - Littérature - Enseignement $22 »)$.
Crédits photographiques: François-Xavier Ferrari.

Bischofberger, B. (dir.) 1995 Ettore Sottsass, Ceramics, Londres: Thames and Hudson.

Bontems, V. 2011 «Le "Cycle de l'image" selon Gilbert Simondon. Une définition génétique de l'image scientifique», Visible 8: 89-104.

Callon, C. 1996 «Le Travail de la conception en architecture», Situations. Les Cahiers de la recherche architecturale $37:$ 25-35. 
Châtelet, G. 1993 Les Enjeux du mobile. Mathématique, physique, philosophie, Paris: Seuil («Des Travaux»).

Deleuze, G. 1986 Foucault, Paris: Éditions de Minuit.

- 1981 Francis Bacon: logique de la sensation, Paris: Éditions de la Différence.

Deleuze, G. \& Guattari, F. 1980 Mille plateaux. Capitalisme et schizophrénie 2, Paris: Éditions de Minuit.

Grandi, D. 2011 [1999] «Ettore Sottsass : sorprese per cui devo cominciare a pensare...» (Entretien avec Ettore Sottsass), Abitare 513. [En ligne] URL: http://www. abitare.it/it/design/ettore-sottsass-sorprese-per-cuidevo-cominciare-a-pensare. Consulté le 30 août 2015.

Guattari, F. 2011 Lignes de fuite. Pour un autre monde de possibles, La Tour d'Aigues: Éditions de l'Aube.

- 1979 L'Inconscient machinique. Essais de schizo-analyse, Paris: Éditions Recherches.

Guiheux, A. dir. 1998 Collection d'architecture du Centre Georges-Pompidou, Paris: Éditions du Centre GeorgesPompidou.

Houdart, S. 2006 «Des multiples manières d'être réel. Les représentations en perspective dans le projet d'architecture», Terrain 46: 107-122.
Houdart, S. \& Minato, C. 2009 Kuma Kengo. Une monographie décalée, Paris: Éditions Donner Lieu.

Jones, J. C. 1970 Design Methods: Seeds of human Futures, Londres: Wiley-Interscience.

Knoespel, K. J. 2004 «Diagrammes, matérialité et cognition», in N. Batt (dir.) Penser par le diagramme de Gilles Deleuze à Gilles Châtelet, Paris: Presses universitaires de Vincennes («Théorie-Littérature-Enseignement 22»): 143-163.

Latour, B. \& Woolgar, S. 1979 Laboratory Life: the Social Construction of Scientific Facts, Beverly Hills: Sage Publications.

Lynch, M. 1993 Scientific Practice and Ordinary Action: Ethnomethodology and Social Studies of Science, Cambridge: Cambridge University Press.

Peirce, C. S. 1978 Écrits sur le signe, Paris: Seuil.

Simondon, G. 2008 Imagination et invention (1965-1966), Chatou: La Transparence.

Yaneva, A. 2009a The Making of a Building. A pragmatist Approach to Architecture, Oxford: Verlag Peter Lang.

— 2009b Made by the Office for Metropolitan Architecture: An Ethnography of Design, Oxford: Verlag Peter Lang.

\section{Pour citer cet article}

Beaubois, V. 2015 «Ethnographies du design. Vers une diagrammatisation de la conception», Techniques\&Culture $n^{\circ} 64$ «Essais de bricologie. Ethnologie de l'art et du design contemporains», p. 48-63. 
